\title{
Existence of exocytotic hemifusion intermediates with a life time up to seconds in type II pneumocytes
}

Pika Miklavc*, Susanne Albrecht*, Oliver H. Wittekindt*, Peter Schullian†, Thomas Haller† and Paul Dietl*

* Institute of General Physiology, University of Ulm, Albert-Einstein Allee 11, Ulm, D89081, Germany

$\dagger$ Department of Physiology and Medical Physics, Medical University of Innsbruck,

Fritz-Pregl-Strasse 3, Innsbruck, A-6020, Austria

Corresponding author:

Dr. Paul Dietl

Professor of Physiology

University of Ulm, Institute of General Physiology

Albert-Einstein-Allee 11

$89081 \mathrm{Ulm}$

Germany

Tel: $+49-731-50023230$

Fax: $+49-731-50023242$

Mob: $+49-176-21643658$

http://WwW. uni-ulm.de/med/allgphys.html

e-mail: paul.dietl@uni-ulm.de

Short title: exocytotic hemifusion in pneumocytes

Keywords: hemifusion, fusion, pneumocyte, lung, darkfield and fluorescence microscopy 


\section{SYNOPSIS}

Exocytosis proceeds through prefusion stages such as hemifusion, but hemifusion is still an elusive intermediate of unknown duration. Using darkfield and fluorescence microscopy in alveolar type II (ATII) cells containing large secretory vesicles ("lamellar bodies", LBs), we show that exocytotic fusion events were accompanied by a mostly biphasic scattered (darkfield) light intensity decrease (SLID) originating from the vesicle border. Correlation with the diffusional behavior of fluorescence markers for either content or membrane mixing revealed that the onset of the fast, second, phase of SLID corresponded to fusion pore formation, which was followed by vesicle swelling. In contrast, a slow, first, phase of SLID preceded pore formation considerably but could still be accompanied by diffusion of farnesylated DsRed, an inner plasma membrane leaflet marker, or nile red. We conclude that hemifusion is an exocytotic intermediate that may last for several seconds. SLID is a new, non-invasive approach by which a prefusion phase, including hemifusion, can be continuously recorded and distinguished from fusion pore formation and postfusion vesicle swelling. 


\section{INTRODUCTION}

Regulated exocytosis denotes stimulated fusion between the limiting membrane of a storage vesicle and the plasma membrane, catalyzed by SNARE proteins under the influence of cytoplasmic factors such as $\mathrm{Ca}^{2+}[1,5,8,12,24,35]$. The fusion pore is the initial aqueous connection between the vesicle lumen and the extracellular space [6]. The initial fusion pore exhibits a channel-like behavior that may flicker between closed and open states, allowing partial release of stored materials $[2,4,14,41]$. The diffusion of hydrophilic compounds through the pore can be preceded by the diffusion of amphiphilic and outer plasma membrane probes, suggesting that the initial lipidic pore is too narrow to enable passage of solutes [28,41]. Models of proteinaceous exocytotic pores have also been proposed [23].

In contrast to fusion pore formation, exocytotic pre- and hemifusion states are still much more refractory to experimental detection. Knowledge thereof is largely based on theoretical considerations, suggesting the "stalk model" as a relatively low energy cost intermediate that initiates hemifusion, through which fusion occurs ("fusion-throughhemifusion", reviewed in detail in [10,48]). Hemifusion is defined as the fusion between two opposing membrane leaflets, enabling diffusion of lipids and probably lipid-anchored proteins from one membrane to the other. Several studies on content vs. lipid mixing convincingly demonstrated the existence of hemifusion in viral and other models of fusion [20,26,27]. In pure phospholipid bilayers, hemifusion was also postulated from electrophysiological evidence [7]. However, hemifusion has not yet been demonstrated as an intermediate stage of exocytosis in a physiological setting, i.e. in an intact, secreting cell. A FRAP study on sea urchin eggs concluded that docked vesicles may be kept stably in a hemifused state by immobile SNARE proteins, but a transition between these stages, i.e. fusion-through-hemifusion, was not directly demonstrated [45]. Likewise, an electron tomography study on synaptic vesicles suggested docked vesicles to be in a hemifused state [47]. Docking and other processes, such as priming, that make a vesicle fusioncompetent, are considered to occur prior to hemifusion and may represent situations where a vesicle is spatially very close to the plasma membrane despite the lack of a lipid connection between both membranes.

Strictly speaking, hemifusion is a morphological description, denoting a certain arrangement of membrane leaflets. However, due to methodological impediments to prove this condition, recent studies have used a more functional description, meaning the exchange of lipid-anchored components between opposing membranes [20,29,45]. Although this functional definition is certainly less accurate in a structural sense, it probably reflects the physiological implications of hemifusion (e.g. exchange of signaling molecules) better than the morphological one. Therefore, we use the term "hemifusion" here in its functional sense, bearing in mind the limitations to predict a clear structural state.

Major impediments to study exocytotic hemifusion are the extremely small diffusion distances that represent a methodological challenge to detect small time lapses between content and membrane mixing. Here we overcome these impediments by studying a cell model (the ATII pneumocyte) which is particularly suitable for the following reasons:

1. LBs are large secretory vesicles with more than $1 \mu \mathrm{m}$ diameter. 
2. LBs contain a surface active complex of lipids and proteins (surfactant), which can be selectively stained by FM 1-43, a membrane-impermeable amphiphilic dye [3,22]. Thereby, content mixing, i.e. the mixing of the extracellular fluid with vesicle contents, elicits a bright localized signal. This readout enables a high temporal resolution of fusion pore formation, as revealed by combined patch clamp (capacitance) and fluorescence experiments [30].

3. In a previous report we showed that single LB fusion events were followed by actin coating of the fused LB, which was found to be a prerequisite for release [33]. Since the fusion pores in these cells open slowly [21] and remain a mechanical barrier for release [40], the shape of a fused LB is maintained for several seconds until the actin coat contracts, facilitating analysis of postfusion membrane mixing. In addition, surfactant is essentially insoluble in water, remaining in a compact form within the fused vesicle before active extrusion.

4. LB exocytosis is slow, sequential, and can be stimulated, facilitating analysis of single fusion events [18].

Here we present the first dynamic resolution of exocytotic prefusion stages including hemifusion and introduce darkfield microscopy as a direct and highly sensitive optical approach to distinguish between pre- and postfusion stages of exocytosis. 


\section{EXPERIMENTAL}

\section{Cell isolation and treatment}

ATII cells were isolated from male Sprague-Dawley rats (150-200 g) as described [22]. In short, rats were anesthetized with ketamin (10\%) and xylazil (2\%). Lungs were perfused, removed from the body, washed and incubated two times with elastase and trypsin at $37^{\circ} \mathrm{C}$ for 15 minutes. Lungs were immersed in DNase containing solution and minced with scissors into bits of approximately $1 \mathrm{~mm}$ size. Enzyme reaction was stopped by incubation with FCS and the tissue was filtered 3 times through gauze and nylon meshes (mesh width: 150, 20, and $10 \mu \mathrm{m}$ ). The final filtrate was centrifuged for $8 \mathrm{~min}$ at $130 \mathrm{x} \mathrm{g}$, the cells were suspended in DMEM medium, and put on IgG coated plastic dishes for $15 \mathrm{~min}$ at $37^{\circ} \mathrm{C}$. Non-adherent cells were centrifuged for $8 \mathrm{~min}$ at $130 \mathrm{x} \mathrm{g}$, suspended in DMEM medium with $10 \%$ FCS and 1\% Penicillin/Streptomycin $(10.000$ units $/ \mathrm{ml}$ Penicillin, $10 \mathrm{mg} / \mathrm{ml}$ Streptomycin) and seeded on glass cover slips. ATII cells were cultured at $37^{\circ} \mathrm{C}, 5 \% \mathrm{CO}_{2}$, and $95 \%$ humidity, and used for experiments $1-2$ days after the isolation. In Lamp3-GFP and DsRed experiments, cells were transfected with adenovirus constructs after isolation. The composition of the experimental solution used for live cell imaging was (in mmol/l): $140 \mathrm{NaCl}, 5 \mathrm{KCl}, 1 \mathrm{MgCl}_{2}, 2 \mathrm{CaCl}_{2}, 5$ glucose, 10 Hepes; $\mathrm{pH}$ 7.4. LB secretion was stimulated with ATP $(100 \mu \mathrm{mol} / \mathrm{l})$. LB fusions were detected with FM 1-43 (300 nmol/l) added to the bath, as described earlier [22]. In the nile red experiments the cells were stained with $200 \mathrm{nmol} / \mathrm{l}$ nile red before the experiment, in DiI experiments the cells were incubated with $1 \mu \mathrm{mol} / 1 \mathrm{DiI}$ for 10 minutes, and in LTR experiments the cells were incubated with $100 \mathrm{nmol} / \mathrm{l}$ LTR for 10 minutes. All dyes were purchased from Molecular Probes. The experiments were made at room temperature $\left(21-25^{\circ} \mathrm{C}\right)$.

\section{Farnesylated DsRed and Lamp3-GFP adenovirus vectors}

Lamp3-GFP adenovirus vector, cloning and cell transfection were recently described [33]. Vectors pDsRed-Monomer-F and pEGFP1-N1 were obtained from Clontech (SaintGermaine-en-Laye, France). Vectors pDONR221 and pAd/CMV/V5-DEST were purchased from Invitrogen (Karlsruhe, Germany).

cDNA encoding DsRed-Farn were amplified from the vector pDsRed-Monomer-F using the primers

DsRed-Farn-F2 (ggggacaagtttgtacaaaaagcaggcttcGTCAGATCCGCTAGCGCTACCGG) and DsRedFarn-R2 (ggggaccactttgtacaagaaagctgggtcAGCTTGAGCTCGAGATCTGGATCC) using standard PCR methods. After isolation of PCR products, amplified cDNA were introduced into pDONR221 by in-vitro recombination using BP-clonase II enzyme mix (Invitrogen, Karlsruhe, Germany) and cloned in E. coli OmniMax ${ }^{\mathrm{TM}} 2-\mathrm{T} 1^{\mathrm{R}}$ (Invitrogen, Karlsruhe, Germany). Thereafter, DsRed-Farn cDNA was transferred into adenoviral expression vector $\mathrm{pAd} / \mathrm{CMV} / \mathrm{V} 5-\mathrm{DEST}$ by in-vitro recombination using LR-clonase II enzyme mix (Invitrogen, Karlsruhe, Germany) and recombination products were cloned in E. coli OmniMax ${ }^{\mathrm{TM}} 2-\mathrm{T}^{\mathrm{R}}$ (Invitrogen, Karlsruhe, Germany).

Multi cloning site (MCS) and coding region of EGFP were amplified from plasmid pEGFP-N1 using primers (GGGGACAAGTTTGTACAAAAAAGCAGGCTATGCTAGCGCTACCGGACTCAG) and

MCSgfp-R1 
(GGGGACCACTTTGTACAAGAAAGCTGGGTATTTACTTGTACAGCTCGTCC) by standard PCR techniques. PCR products were introduced into pDONR221 by in-vitro recombination using BP-clonase II enzyme mix (Invitrogen, Karlsruhe, Germany) and cloned in E. coli OmniMax ${ }^{\mathrm{TM}} 2-\mathrm{T} 1^{\mathrm{R}}$ (Invitrogen, Karlsruhe, Germany).

Recombinant adenoviral expression vectors were isolated from E.coli cells using QIAGEN plasmid midi kit (Qiagen, Hilden, Germany). Plasmids were linearised using restriction endo-nucleases PacI (New England Biolabs, Frankfurt, Germany). Linearised plasmid was transfected into 293A cells (Invitrogen, Karlsruhe, Germany) using Lipofectamine 2000 (Invitrogen, Karlsruhe, Germany) without further purification directly after heat inactivation of PacI. Transfected cells were cultivated for 5 to 8 days and virus particles were isolated using ViraBind Adenovirus purification kit (Cell Biolabs, Heidelberg, Germany) according to manufacturers' protocol. Virus particles were eluted in elution buffer (Cell Biolabs, Heidelberg, Germany), and stored in elution buffer containing $10 \%$ glycerol in aliquots at $-80^{\circ} \mathrm{C}$ until use.

\section{Imaging with the RTM 2.5 microscope}

Darkfield and fluorescence imaging were performed on an upright Richardson RTM 2.5 microscope (Improvision, Germany). For darkfield illumination we used the red or the blue filter in the condenser; therefore the light-reflecting structures appeared red or blue on a dark background. Darkfield and fluorescence light was detected with a 3CCD color video camera (ExwaveHAD DXC-C33, Sony). Images were acquired with the $63 \mathrm{x}$ water immersion objective (Leica) and the RTM 3.0 blue filter cube (490 nm dichroic, 400-500 $\mathrm{nm}$ excitation filter, 510 LP emission filter) for FM 1-43, Lamp3-GFP, and CTG imaging, and with the RTM 3.0 green filter cube $(560 \mathrm{~nm}$ dichroic, $540-550 \mathrm{~nm}$ excitation filter, 570 LP emission filter) for farnesylated DsRed, DiI, LTR, and nile red imaging. Volocity software was used for image acquisition and subsequent measurements. At the image acquisition rate of 5-10/s the darkfield and fluorescence signals were acquired simultaneously and were subsequently digitally separated from an RGB image based on their different color. In combined darkfield and FM 1-43 fluorescence experiments we used the red channel for darkfield and green channel for FM 1-43 fluorescence signals, and in combined darkfield and farnesylated DsRed, DiI and nile red experiments we used the blue channel for darkfield and red channel for fluorescence signals.

\section{Imaging with the fluorescence microscope}

The combined LTG and nile red measurements were performed with a 100x Neofluar oil, NA 1.3 on a Zeiss Axiovert equipped for epifluorescence (Polychrome II, TILL Photonics), emission beam splitting (DV-CC, Optical Insights LLC) and synchronous image acquisition (Sensicam, PCO) at a rate of $1 / \mathrm{s}$. Wavelength and filters used were: $485 \mathrm{~nm}$ exc., $520 \mathrm{~nm}$ dichroic 1, $565 \mathrm{~nm}$ dichroic 2, $525 \mathrm{~nm}$ BP (LTG) and $630 \mathrm{~nm}$ LP (nile red). Cells were pre-incubated $30 \mathrm{~min}$ with LTG, $200 \mathrm{~nm}$, nile red was added at 4 $\mu \mathrm{mol} / 15 \mathrm{~min}$ prior to ATP, $50 \mu \mathrm{mol} / 1$.

\section{Image and statistical analysis}

A square region of interest was defined around the fusing LB, on which darkfield light and fluorescence intensity were measured. In darkfield and fluorescence measurements 
the lowest value was subtracted and the change in light intensity was expressed in arbitrary units. Unless otherwise stated, data are presented as mean \pm s.d. Vesicle size was measured as the surface of the vesicle intersection in one focal plane. Vesicle volume change after fusion was calculated from the measured vesicle intersection in one focal plane, and median and interquartile ranges were calculated. Tau values were obtained by fitting the curve with a single exponential in Igor Pro 5.04B. Excel and SigmaPlot 10.0 were used for statistics and graphs. Some images were corrected for brightness and contrast with Adobe Photoshop CS2. 


\section{RESULTS}

Measurement of single LB fusion with the plasma membrane by darkfield microscopy and FM 1-43 fluorescence was recently described in detail [33]. It exhibits the following key feature: The darkfield light intensity originating from the border of a fusing LB decreases (SLID), while vesicle contents appear dark (Figure 1). When FM 1-43 is present in the bath, this is accompanied by an increase of FM 1-43 fluorescence ( $\left.F_{F M 1-43}\right)$ within the fused LB, starting at the site of the fusion pore (Figure 1). $\mathrm{F}_{\mathrm{FM} 1-43}$ originating from surfactant exceeds outer plasma membrane staining so manifold that the plasma membrane appears virtually dark (Figure 1).

To further investigate the obvious correlation between SLID and the fusion pore opening, we used higher image acquisition rates $(10 / \mathrm{s})$. These measurements revealed that SLID consists of two clearly discernible stages (Figure 2) - a slow component at the beginning (mean duration 2.24 s; s.d. $=2.19, \mathrm{n}=22$ ) and a subsequent fast one (mean duration 0.92 s; s.d. $=0.49)$. The onsets of the two phases of SLID were measured as shown in Figure 2. The linear parts of the darkfield data were fitted and the crossing points of the linear regressions were considered as onsets of SLID phase 1 and 2 . The onset of the $\mathrm{F}_{\mathrm{FM} 1-43}$ increase (indicating fusion pore opening [30]) corresponded to the start of the fast component of SLID (Figure 3). The highest observed discrepancy between the two events was $0.5 \mathrm{~s}$. Therefore, it is reasonable to assume that the light intensity decrease gets faster when the fusion pore opens (either transiently or permanently). This assumption leads to the question what the initial slow component of SLID might reflect. Despite its variable duration, it was detectable in the majority (84.6\%) of the observed fusion events. Theoretically, several explanations for the biphasic nature of SLID are possible, which will be discussed below. The most likely one is that the first phase of SLID reports the hemifusion stage ("membrane mixing"), where the proximal leaflets of the plasma and vesicle membranes merge but the fusion pore is not yet open. During this stage, a non membrane-permeable dye like FM 1-43 would not be able to enter the LB. The second phase of SLID corresponds to an open fusion pore and "content mixing".

To further test the hypotheses that hemifusion intermediates of considerable duration indeed exist in type II cells, we sought several independent experimental approaches, applying markers for either content or membrane mixing, as illustrated in Figure 4A: DiI was used as a marker of the outer plasma membrane leaflet [39]. In contrast to FM 1-43, it was absent in the bath solution. Therefore, LB staining was exclusively due to lateral membrane diffusion, but not to bulk fluid entry. Conversely, LysoTracker Green (LTG) or LysoTracker Red (LTR) were used as fluid phase content markers. They are trapped in LBs by non-ionic diffusion and protonation, and rapidly discharged into the extracellular space upon the formation of an aqueous pore [22]. The onset of LTG discharge coincides $(<1 \mathrm{~s})$ with uptake of FM 1-43 [22] and also with the dissipation of the proton gradient, the probably earliest diffusional event within the lumen of the pore (see supplementary data Figure 1). To probe for diffusion of components between the leaflets of the inner plasma membrane and outer LB membrane (hemifusion), we used two different dyes: First, farnesylated DsRed which selectively incorporates into the inner leaflet of the plasma membrane by posttranslational farnesylation of the protein through membranebound enzymes [19]. Under the assumption that the hemifusion stalk does not contain diffusion barriers for this dye, it should diffuse from the inner leaflet of the plasma 
membrane to the outer leaflet of the LB membrane during hemifusion. Second, the unspecific lipid marker nile red, which is trapped within phospholipids [17], resulting in stable staining of LBs (Figures 4 and 6). In the course of hemifusion, the LB should lose nile red fluorescence intensity, possibly due to dye redistribution along the plasma membrane.

Figure 4A exhibits the fluorescence stainings of individual LBs before and after both phases of SLID, i.e. the initial pre- and final postfusion stages. It illustrates that all tested dyes behaved according to the theoretical predictions outlined above with certain limitations regarding DsRed, which exhibited a wide scatter in its diffusional behavior (see below, Figure 5A, B and Discussion). In the absence of SLID, the fluorescence intensities of these markers were stable or subject to variable extents of photobleaching.

A direct comparison of the earliest onsets of fluorescence changes between these dyes is shown in Figure 4B: DiI and LTR behaved like FM 1-43, exhibiting fluorescence change during phase 2 of SLID exclusively. The onset of DsRed and nile red fluorescence change, however, could start as early as the beginning of phase 1 of SLID (Figure 4B). Hence, the earliest observed onsets of fluorescence changes by markers of lipid vs. content mixing coincided almost precisely with the onsets of phase 1 and phase 2 of SLID, respectively.

Assuming the onset of the second, fast, phase of SLID as a marker for the instance of fusion pore formation, Figure 5A exhibits the temporal relation (negative values represent prefusion events) between all fluorescence and SLID phase 1 changes with reference to this event $(=$ time 0$)$. It is again apparent that the diffusion of content / outer leaflet markers (LTR, DiI, FM 1-43) followed the fusion pore formation. In contrast, nile red and DsRed fluorescence changes could start several seconds before fusion. There was, on average, a delay between the onset of SLID phase 1 and particularly that of DsRed, which could extend into the postfusion phase (Figure 5A). Hence, analysis of hemifusion by specific membrane diffusion probes is considerably limited by highly variable diffusion times and/or limitations of detection, as will be discussed below.

To further analyze a possible relation between SLID phase 1 and membrane merger (hemifusion), we plotted individual fluorescence changes as relative delays following the onset of SLID phase 1, shown in Figure 5B. The data reveal that although there was a clear tendency of nile red and DsRed to lag behind the onset of SLID phase 1, some events closely coincided (Figure 4B). Importantly, however, a fluorescence change was never observed before SLID phase 1. Hence, hemifusion cannot precede SLID phase 1.

To obtain a direct comparison between lipid and content mixing within a single vesicle, we used a combination of nile red and LTG. As indicated above, both dyes accumulate within LBs (Figure 6A). The spectral emission properties of both dyes allowed their synchronous recording with a beam splitting device in the emission path. Figure 6B demonstrates that the decline in nile red intensity precedes that of LTG $(1.36 \mathrm{~s}$, s.d. $=0.8$, $\mathrm{n}=7$ ). Also here, the most reasonable explanation is a situation where vesicle and plasma membrane are physically conjunct, seconds before a water permeable pore is formed.

The concept of hemifusion vs. fusion was further examined by analyzing vesicle swelling (Figure 7A). Postfusion swelling is a common finding, driven by fluid uptake through the fusion pore due to the physico-chemical properties of the vesicle matrix [16,32]. Postfusion swelling can therefore be taken as another parameter of "content mixing", independent of content marker diffusion. Fusion pore formation (measured as the start of 
FM 1-43 staining) was followed by swelling of LBs (Figure 7B), measured as the surface change of the scattered light signal. Prefusion swelling, as reported in another cell type [25], was never observed here. The median increase of the LB volume estimated from darkfield images was $17.5 \%$ (interquartile range $5.8-24.3 \%, \mathrm{n}=50$ ). Postfusion LB swelling occurred within seconds and was therefore several fold faster than the initiation of release [22,33], (time constant of postfusion swelling: $\tau=2.1$ s, s.d. $=1.4, n=6$ ). To exclude the possibility that the diameter increase of the scattered light signal was just the result of a change in light diffraction properties, we transfected the ATI cells with Lamp3-GFP fusion protein. Lamp3 is localized at the membrane of lysosome associated organelles, and in ATII cells the Lamp3-GFP fluorescence and the darkfield signal overlap [33]. As in the darkfield illumination, the swelling was also detectable by measuring the Lamp3-GFP signal (Figure 7C; median increase in volume 30.9\%, interquartile range $18.5-38.4 \%, \mathrm{n}=5$ ). Additional evidence for LB swelling is presented in supplementary data Figure 2.

These experiments indicate that content markers or water can enter the LB during the second phase of SLID exclusively, which makes this phase a sensitive postfusion indicator. The DsRed and nile red experiments indicate the existence of hemifusion intermediates that may last for seconds. SLID phase 1 is a newly described prefusion indicator that may represent the hemifusion state even more reliably and precisely than any diffusion marker, as discussed in the following. 


\section{DISCUSSION}

Two opposing theories describe initial exocytotic fusion pores as being either proteinaceous (direct fusion pathway), or lipidic, emerging through transient hemifusion states (stalk model) [10]. Our study provides first experimental evidence in favor of the "fusion-through-hemifusion" model in a living, intact cell. It demonstrates prefusion and membrane merger seconds before content mixing in single secretory vesicles. Thereby, it confirms predictions from a multitude of non-exocytotic or artificial fusion systems, reviewed in detail elsewhere $[10,11,48]$. It also supports evidence in favor of "stable" hemifusion states of "docked" vesicles [45,47].

Decades of intensive research, including breath-taking advances in molecular and live cell imaging techniques, have yet failed to develop a method to continuously record exocytotic hemifusion states in living cells. SLID (phase 1) is a new approach in this direction. We are aware that at the time being, SLID is not more than a new phenomenon, but it yields a continuous recording of 2 phases, of which phase 1 can clearly be assigned to a prefusion stage. Its validation as a method that accurately reflects the morphological postulate of the hemifusion state will only be possible when hemifusion will be accessible to independent measurement at all. To this end, electron microscopy would be the method of choice, but this is far beyond the temporal resolution and technical feasibility, providing static information of the process only.

SLID (phase 1) is a prefusion indicator that coincides fairly well, but not precisely, with diffusional membrane merger (Figure 5). Going beyond hemifusion, it could reflect any other conceivable pre-fusion condition, such as protein rearrangement and development of a tighter contact between the vesicle and the plasma membrane. Hence, the key question remains: What does SLID actually report?

Darkfield microscopy has been extensively used to investigate the fusion and transformational properties of giant liposomes [36,37]. These studies suggest that the light scattering intensity from a liposomal membrane reflects its thickness, and that the changes in scattered light intensity of liposomal membranes observed with the darkfield microscope might also be based on the cooperative movement of lipid molecules in a membrane $[36,37]$. We also made the observation that extruded surfactant, in contrast to surfactant within LBs, generated a darkfield signal, probably because the highly organized lipid layers in LBs [38] became disordered. The origin of the darkfield signal from the limiting membrane of an LB is more difficult to interpret: The membrane of a secretory vesicle is not merely an even surface of free-floating phospholipids, but is probably endowed with a highly complex 3D topography due to abundant membraneassociated proteins [42]. Although we do not know the exact composition, distribution and density of these proteins in LBs [44], they may affect the optical properties of the incident light. In any case, it can be assumed that SLID rather reports a change of LB surface properties than of vesicle content, since the first phase of SLID precedes measurable content mixing and LB swelling by seconds.

We have following reasons to favor the hypothesis that SLID phase 1 indeed reports the hemifusion state:

1. Lipid flow explains the biphasic nature of SLID much better than other prefusion processes, since the two phases of SLID would correspond with the two leaflets of the membrane, merging in sequence. In this case, the first phase of SLID would reflect a 
change of lipid composition in the outer leaflet of the limiting LB membrane. Although there is no direct way to test this hypothesis, it is consistent with other observations: First, darkfield microscopy gives the best high-contrast images of giant liposomes compared with other methods, indicating that darkfield images do not require the presence of membrane proteins [36]. Furthermore, detergents like Triton X, which disrupt lipid domains, sequentially caused SLID in all LBs [33]. The lipid composition of a membrane is fundamental in determining its curvature and tension [9]. In this way, specific lipids do not only determine the readiness of a secretory vesicle to hemifuse and fuse, but also create a force for lipids to flow when membrane leaflets merge [13]. Monck et al. demonstrated lipid flow from the plasma to the vesicle membrane during exocytosis, resulting in up to $40 \%$ loss of plasma membrane area [34]. It is likely that the inner plasma membrane leaflet and outer LB membrane leaflet have different lipid compositions [43], which would be sufficient to explain substantial lipid flow during hemifusion.

2. The delay between the onset of SLID phase 1 and the change of DsRed or nile red fluorescence intensity does not exclude that membrane merger is the common underlying mechanism: Free lipid flow through the hemifusion stalk may be limited $[11,48]$. This could explain the observation that phase 1 of SLID does not occur abruptly (as a step), but rather linearly and slowly. If true, the slope of phase 1 would actually reflect diffusional limitations. Accordingly, hemifusion can present a barrier for the diffusion of proteins with the size of farnesylated DsRed [20]. In addition, we have to consider photobleaching (rapid decrease of DsRed fluorescence before and after fusion, Figure 4B) which can also explain the low detectability of a small DsRed increase before the fusion pore opening.

3. The existence of fusion events, where diffusion of farnesylated DsRed into the vesicle almost precisely coincided with the onset of SLID phase 1, indicates that membrane merger and SLID phase 1 can occur simultaneously. Hence, SLID phase 1 does not require any other or additional explanation than hemifusion alone.

4. In theory, both phases of SLID could occur after fusion pore formation, but the initial pore is so small that no "content mixing" (entry of extracellular fluid) would take place. This possibility, however, is very unlikely for the following reasons: First, we showed in simultaneous patch-clamp and FM 1-43 measurements that the fusion pore opening (measured as capacitance steps) and the increase in $\mathrm{F}_{\mathrm{FM} 1-43}$ are apart by no more than $1 \mathrm{~s}$ [30], which is considerably shorter than the duration of the first phase of SLID (2.24 s on average, $9 \mathrm{~s}$ maximum). Second, even if such "narrow pores" existed (small enough to escape detection by capacitance measurements), FM 1-43 should be able to enter the LB along the wall of the fusion pore, by lateral diffusion from the outer leaflet of the plasma membrane [28].

Our experiments revealed that there is no vesicle swelling prior to fusion pore formation. Hence, the prefusion phase was not accompanied by volume uptake. It does not appear, therefore, that vesicle swelling in ATII cells is a driving force for fusion pore formation $[16,25]$.

Independent of the prefusion marker (SLID phase 1, nile red, or DsRed), the hemifusion could last longer then 4 seconds (Figure 5A). Hence, considering the large variability in the duration of individual hemifusion states (not detectable to $9 \mathrm{~s}$ ), these different methods yielded fairly consistent results. They suggest that hemifusion lifetimes cannot 
be solely explained on the basis of a different vesicle size. It rather appears that hemifusion does not proceed in a stereotypic way, but that specific lipid or protein domains in opposing membranes determine the transition to fusion. This prompts the question if hemifusion is an actively regulated process and which compounds are involved therein. It also prompts the question if transient hemifusion and LB retrieval exist in addition to kiss-and-run events [33]. In any case, the duration of the hemifusion state should influence the amount of membrane mixing. This may induce translocation of plasma membrane signaling molecules to secretory vesicles, triggering signaling cascades that induce - among others - actin assembly and influence the future mobility of the fused vesicle $[31,33,46]$. In the lung, these processes may determine polarized secretion, compound exocytosis and membrane recycling [15].

The potential of darkfield microscopy will be to disclose regulatory principles of hemifusion and possibly other prefusion events that are inaccessible by fluorescence probes, which are all subject to limitations caused by diffusion properties, photobleaching, staining specificity or spectral features. 


\section{FUNDING AND ACKNOWLEDGMENTS}

This work was supported by the Fonds zur Förderung der Wissenschaftlichen Forschung [P15742, P15743 and P20472], the Deutsche Forschungsgemeinschaft [D1402], and the 6th framework of the European Union, Pulmo-Net. Technical assistance by Melanie Timmler, Tatiana Felder, Gerlinde Siber and Irina Öttl is gratefully acknowledged. 


\section{ABBREVIATIONS LIST}

ATII, alveolar type II; F F1-43, FM 1-43 fluorescence intensity; LB, lamellar body; SLID, scattered light intensity decrease. 


\section{REFERENCES}

1 Almers, W. (1990) Exocytosis. Annu. Rev. Physiol., 52, 607-624

2 Alvarez de Toledo, G., Fernandez-Chacon, R. and Fernandez, J. M. (1993) Release of secretory products during transient vesicle fusion. Nature, 363, 554-558

3 Betz, W. J. and Bewick, G. S. (1992) Optical analysis of synaptic vesicle recycling at the frog neuromuscular junction. Science, 255, 200-203

4 Breckenridge, L. J. and Almers, W. (1987) Final steps in exocytosis observed in a cell with giant secretory granules. Proc. Natl. Acad. Sci. USA, 84, 1945-1949

5 Burgoyne, R. D. and Morgan, A. (2002) Secretory granule exocytosis. Physiol. Rev., 83, 581-632

6 Chandler, D. E. and Heuser, J. E. (1980) Arrest of membrane fusion events in mast cells by quick-freezing. J. Cell Biol., 86, 666-674

7 Chanturiya, A., Chernomordik, L. V. and Zimmerberg, J.(1997) Flickering fusion pores comparable with initial exocytotic pores occur in protein-free phospholipid bilayers. Proc. Natl. Acad. Sci. USA, 94, 14423-14428

8 Chen, Y. A. and Scheller, R. H. (2001) SNARE-mediated membrane fusion. Nat. Rev. Mol. Cell Biol., 2, 98-106

9 Chernomordik, L., Chanturiya, A., Green, J. and Zimmerberg, J. (1995) The hemifusion intermediate and its conversion to complete fusion: regulation by membrane composition. Biophys. J, 69, 922-929

10 Chernomordik, L. V. and Kozlov, M. M. (2005) Membrane hemifusion: crossing a chasm in two leaps. Cell, 123, 375-382

11 Chernomordik, L. V. and Kozlov, M. M. (2008) Mechanics of membrane fusion. Nat. Struct. Mol. Biol., 15, 675-683

12 Chieregatti, E. and Meldolesi, J. (2005) Regulated exocytosis: new organelles for nonsecretory purposes. Nat. Rev. Mol. Cell Biol., 6, 181-187

13 Chizmadzhev, Y. A., Kuzmin, P. I., Kumenko, D. A., Zimmerberg, J. and Cohen, F. S. (2000) Dynamics of fusion pores connecting membranes of different tensions. Biophys. J., 78, 2241-2256

14 Chow, R. H., von Ruden, L. and Neher, E. (1992) Delay in vesicle fusion revealed by electrochemical monitoring of single secretory events in adrenal chromaffin cells.

Nature, 356, 60-63 
15 Dietl, P. and Haller, T. (2005) Exocytosis of lung surfactant: from the secretory vesicle to the air-liquid interface. Annu. Rev. Physiol., 67, 595-621

16 Finkelstein, A., Zimmerberg, J. and Cohen, F. S. (1986) Osmotic swelling of vesicles: its role in the fusion of vesicles with planar phospholipid bilayer membranes and its possible role in exocytosis. Annu. Rev. Physiol, 48, 163-174

17 Fowler, S. D., Brown, W. J., Warfel, J. and Greenspan, P. (1987) Use of nile red for the rapid in situ quantitation of lipids on thin-layer chromatograms. J. Lipid Res., 28, $1225-1232$

18 Frick, M., Eschertzhuber, S., Haller, T., Mair, N. and Dietl, P. (2001) Secretion in alveolar type II cells at the interface of constitutive and regulated exocytosis. Am. J. Respir. Cell Mol. Biol., 25, 306-315

19 Fujiyama, A., Tsunasawa, S., Tamanoi, F. and Sakiyama, F. (1991) S-farnesylation and methyl esterification of C-terminal domain of yeast RAS2 protein prior to fatty acid acylation. J. Biol. Chem., 266, 17926-17931

20 Giraudo, C. G., Hu, C., You, D., Slovic, A. M., Mosharov, E. V., Sulzer, D., Melia, T. J. and Rothman, J. E. (2005) SNAREs can promote complete fusion and hemifusion as alternative outcomes. J. Cell Biol., 170, 249-260

21 Haller, T., Dietl, P., Pfaller, K., Frick, M., Mair, N., Paulmichl, M., Hess, M. W., Furst, J. and Maly, K. (2001) Fusion pore expansion is a slow, discontinuous, and $\mathrm{Ca}^{2+}$ dependent process regulating secretion from alveolar type II cells. J. Cell Biol., 155, 279-289

22 Haller, T., Ortmayr, J., Friedrich, F., Volk1, H. and Dietl, P. (1998) Dynamics of surfactant release in alveolar type II cells. Proc. Natl. Acad. Sci. USA, 95, 1579-1584

23 Han, X., Wang, C. T., Bai, J., Chapman, E. R. and Jackson, M. B. (2004)

Transmembrane segments of syntaxin line the fusion pore of $\mathrm{Ca}^{2+}$-triggered exocytosis.

Science, 304, 289-292

24 Jahn, R., Lang, T. and Südhof, T. C. (2003) Membrane fusion. Cell, 112, 519-533

25 Jena, B. P., Schneider, S. W., Geibel, J. P., Webster, P., Oberleithner, H. and Sritharan, K. C. (1997) Gi regulation of secretory vesicle swelling examined by atomic force microscopy. Proc. Natl. Acad. Sci. USA, 94, 13317-13322

26 Jun, Y. and Wickner, W. (2007) Assays of vacuole fusion resolve the stages of docking, lipid mixing, and content mixing. Proc. Natl. Acad. Sci. USA, 104, 1301013015

27 Kemble, G. W., Danieli, T. and White, J. M. (1994) Lipid-anchored influenza hemagglutinin promotes hemifusion, not complete fusion. Cell, 76, 383-391 
28 Klauke, N. and Plattner, H. (2000) "Frustrated Exocytosis"--a novel phenomenon: membrane fusion without contents release, followed by detachment and reattachment of dense core vesicles in Paramecium cells. J. Membr. Biol., 176, 237-248

29 Lu, X., Zhang, F., McNew, J. A. and Shin, Y. K. (2005) Membrane fusion induced by neuronal SNAREs transits through hemifusion. J. Biol. Chem., 280, 30538-30541

30 Mair, N., Haller, T. and Dietl, P. (1999) Exocytosis in alveolar type II cells revealed by cell capacitance and fluorescence measurements. Am. J. Physiol, 276, L376-L382

31 Malacombe, M., Bader, M. F. and Gasman, S. (2006) Exocytosis in neuroendocrine cells: new tasks for actin. Biochim. Biophys. Acta, 1763, 1175-1183

32 Marszalek, P. E., Farrell, B., Verdugo, P. and Fernandez, J. M. (1997) Kinetics of release of serotonin from isolated secretory granules. II. Ion exchange determines the diffusivity of serotonin. Biophys. J., 73, 1169-1183

33 Miklavc, P., Wittekindt, O.H., Felder, E. and Dietl, P. (2009) $\mathrm{Ca}^{2+}$-dependent actin coating of lamellar bodies after exocytotic fusion: a prerequisite for content release or kiss-and-run. Ann. NY Acad. Sci., 1152, 43-52

34 Monck, J. R., Alvarez de Toledo, G. and Fernandez, J. M. (1990) Tension in secretory granule membranes causes extensive membrane transfer through the exocytotic fusion pore. Proc. Natl. Acad. Sci. USA, 87, 7804-7808

35 Neher, E. (1998) Vesicle pools and $\mathrm{Ca}^{2+}$ microdomains: new tools for understanding their roles in neurotransmitter release. Neuron, 20, 389-399

36 Nomura, F., Inaba, T., Ishikawa, S., Nagata, M., Takahashi, S., Hotani, H. and Takiguchi, K. (2004) Microscopic observations reveal that fusogenic peptides induce liposome shrinkage prior to membrane fusion. Proc. Natl. Acad. Sci. USA, 101, 34203425

37 Nomura, F., Nagata, M., Inaba, T., Hiramatsu, H., Hotani, H. and Takiguchi, K. (2001) Capabilities of liposomes for topological transformation. Proc. Natl. Acad. Sci. USA, 98, 2340-2345

38 Perez-Gil, J. (2008) Structure of pulmonary surfactant membranes and films: The role of proteins and lipid-protein interactions. Biochim. Biophys. Acta, 1778, 1676-1695

39 Roddy, T. P., Cannon, D. M., Jr., Ostrowski, S. G., Winograd, N. and Ewing, A. G. (2002) Identification of cellular sections with imaging mass spectrometry following freeze fracture. Anal. Chem., 74, 4020-4026

40 Singer, W., Frick, M., Haller, T., Bernet, S., Ritsch-Marte, M. and Dietl, P. (2003) Mechanical forces impeding exocytotic surfactant release revealed by optical tweezers. Biophys. J, 84, 1344-1351 
41 Takahashi, N., Kishimoto, T., Nemoto, T., Kadowaki, T. and Kasai, H. (2002) Fusion pore dynamics and insulin granule exocytosis in the pancreatic islet. Science, 297, 13491352

42 Takamori, S., Holt, M., Stenius, K., Lemke, E.A., Gronborg, M., Riedel, D., Urlaub, H., Schenck, S., Brugger, B., Ringler, P., Muller, S. A., Rammner, B., Grater, F., Hub, J. S., De Groot, B. L., Mieskes, G., Moriyama, Y., Klingauf, J., Grubmuller, H., Heuser, J., Wieland, F. and Jahn, R. (2006) Molecular anatomy of a trafficking organelle. Cell, 127, 831-846

43 van Meer. G., Voelker, D. R. and Feigenson, G. W. (2008) Membrane lipids: where they are and how they behave. Nat. Rev. Mol Cell Biol., 9, 112-124

44 Wang, P., Chintagari, N. R., Narayanaperumal, J., Ayalew, S., Hartson, S. and Liu, L. (2008) Proteomic analysis of lamellar bodies isolated from rat lungs. BMC. Cell Biol., 9, 34

45 Wong, J. L., Koppel, D. E., Cowan, A. E. and Wessel, G. M. (2007) Membrane hemifusion is a stable intermediate of exocytosis. Dev. Cell, 12, 653-659

$46 \mathrm{Yu}, \mathrm{H}$. Y. and Bement, W. M. (2007) Control of local actin assembly by membrane fusion-dependent compartment mixing. Nat. Cell Biol., 9, 149-159

47 Zampighi, G. A., Zampighi, L. M., Fain, N., Lanzavecchia, S., Simon, S.A. and Wright, E. M. (2006) Conical electron tomography of a chemical synapse: vesicles docked to the active zone are hemi-fused. Biophys. J., 91, 2910-2918

48 Zimmerberg, J. and Chernomordik, L. V. (1999) Membrane fusion. Adv. Drug Deliv. Rev., 38, 197-205 

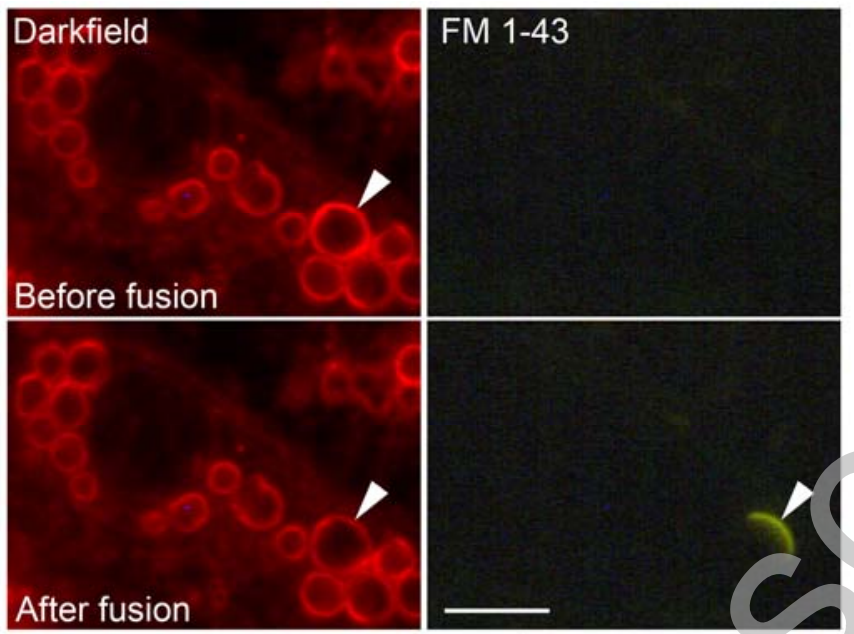

Figure 1: ATII cell imaged with darkfield illumination (left) and FM 1-43 fluorescence (right), before and after fusion (delay $=12$ seconds) of one LB (arrowhead) with plasma membrane. Note that the darkfield light intensity of this vesicle decreased, which was not the case in other vesicles. Note also early staining of LB content with FM 1-43 at the presumptive site of the fusion pore. Scale bar $=8 \mu \mathrm{m}$. 

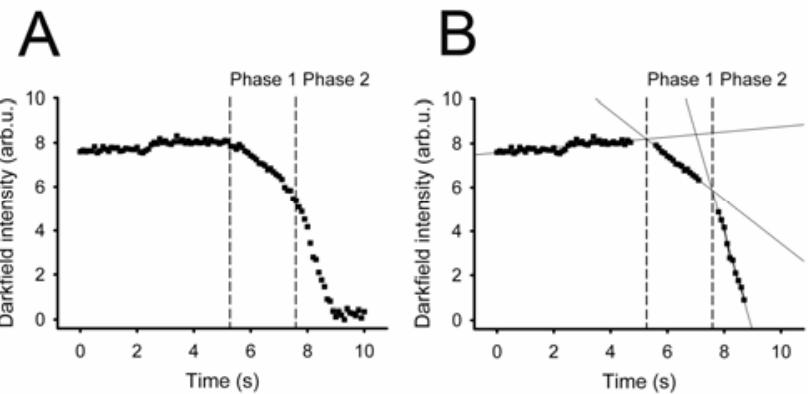

Figure 2: Determination of the times of onset of scattered light intensity decrease (SLID) phase 1 and 2. A. Original darkfield tracing of a lamellar body at the time of fusion. B. Linear parts of the darkfield tracing (selected as shown here for the example in A) were fitted by a least square algorithm, and the crossing points of the linear regression lines were considered as the times of onset of SLID phase 1 and 2, respectively. 


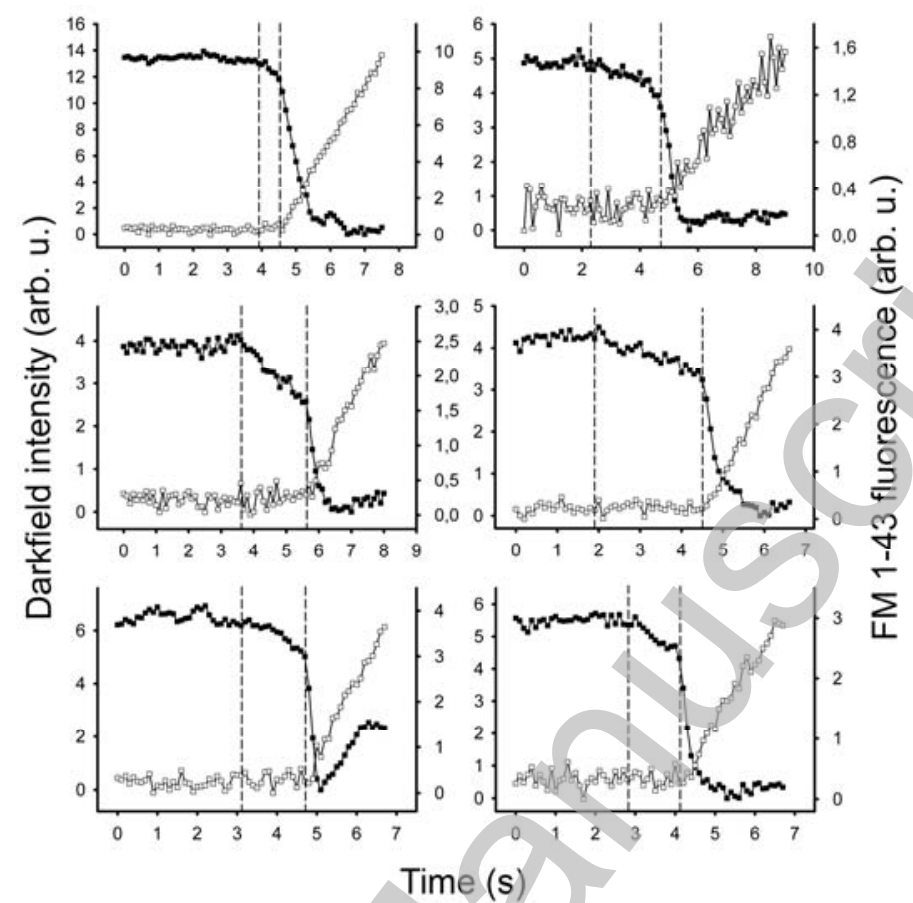

Figure 3: The time courses of darkfield light intensity change (filled squares) and $\mathrm{F}_{\mathrm{FM} 1-43}$ change (open squares) for 6 fusion events imaged with time resolution 10/s. The left dashed line on each graph indicates the start of SLID; the right dashed line indicates the transition of SLID from the first, slow to the second, fast phase. The latter corresponded to the start in FM 1-43 fluorescence increase, indicating fusion pore opening. Lowest light intensity values were set zero. 


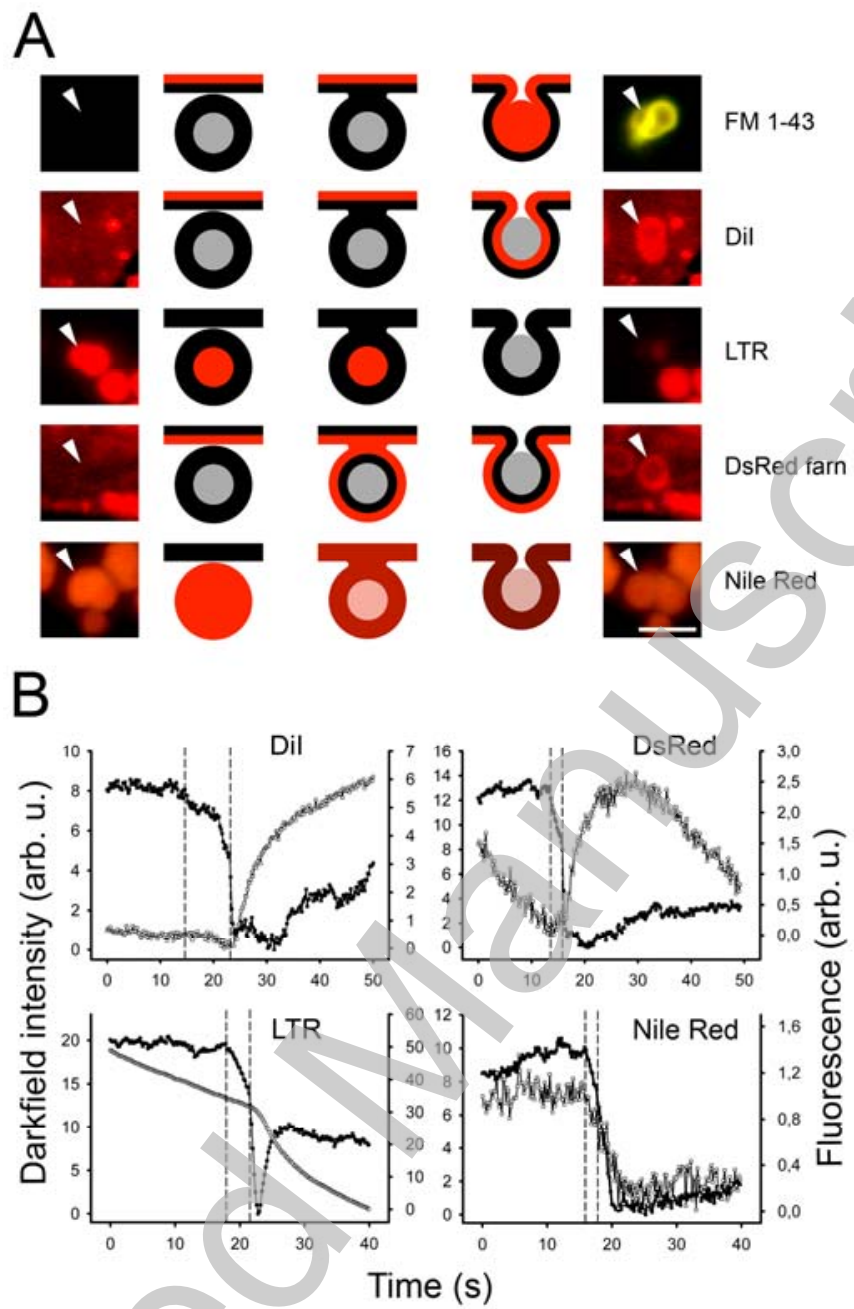

Figure 4: SLID and its relation with fluorescence of membrane / content markers. A. Scheme of LBs in the presence of fluorescence indicators in the pre- (left), hemi(middle) and postfusion (right) stages. Red color indicates postulated site of fluorescence during each stage. Gray circle denotes surfactant. Note that the upper 3 dyes report fusion pore formation, whereas the lower 2 should report lipid merger. Examples of fluorescence images of single LBs before and after fusion with the plasma membrane (arrowheads) are shown on the left and right side, respectively. Scale bar $=4 \mu \mathrm{m}$. B. Examples of earliest onsets of fluorescence changes (open squares) for 4 different dyes in comparison with SLID (filled squares). The time between the two dashed lines indicates SLID phase 1. The prominent decrease in LTR and DsRed fluorescence before and after fusion are due to photobleaching. 


\section{A}

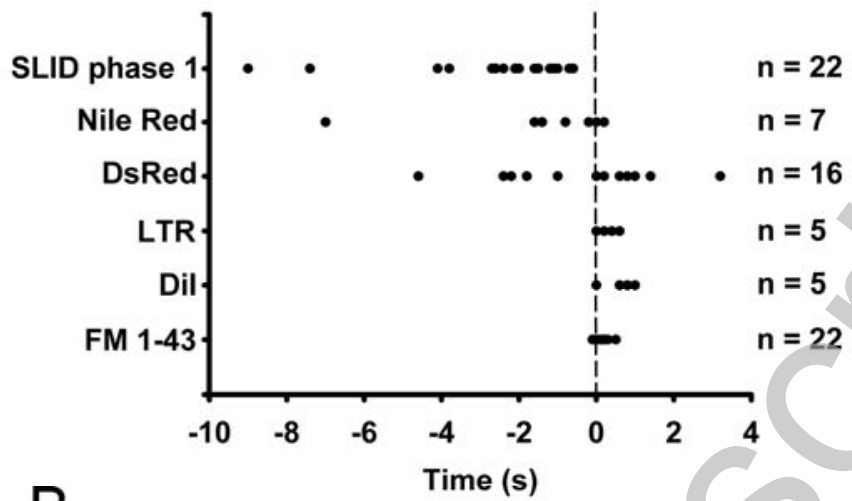

B

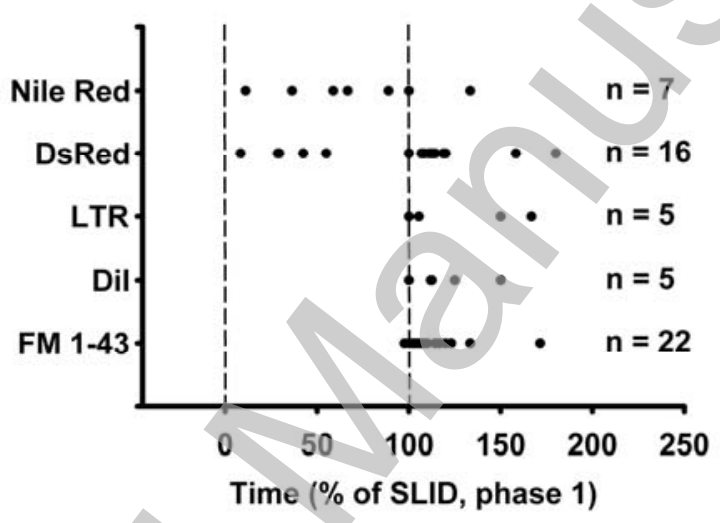

Figure 5: Summary of SLID and fluorescence markers. A. Analysis of the onsets of SLID phase 1 and fluorescence intensity changes (shown as single data points for each fusion event) in comparison to fusion pore formation (defined as onset of SLID phase 2, time $=0$, dashed line; Negative values indicate times before fusion pore formation, positive values times thereafter). B. Analysis of the onsets of fluorescence intensity changes (shown as single data points for each fusion event). Instance of fluorescence change is expressed as percent of SLID phase $1.0-100 \%$ denotes the entire duration of SLID phase 1 (space between dashed lines). Note that DsRed fluorescence exhibited the least precise correlation with a specific phase, which can be explained by restricted probe diffusion through the early hemifusion structures and by photobleaching (see Discussion). 


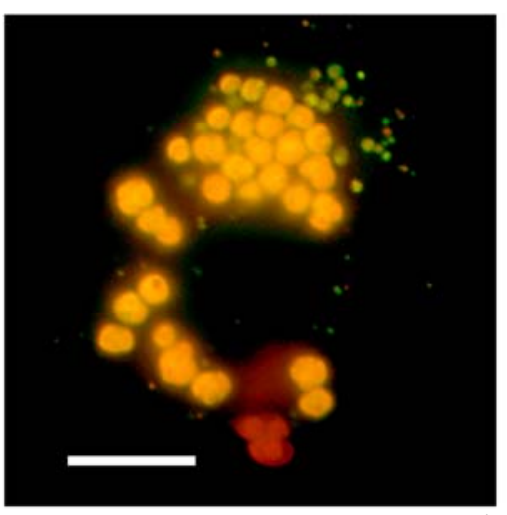

B

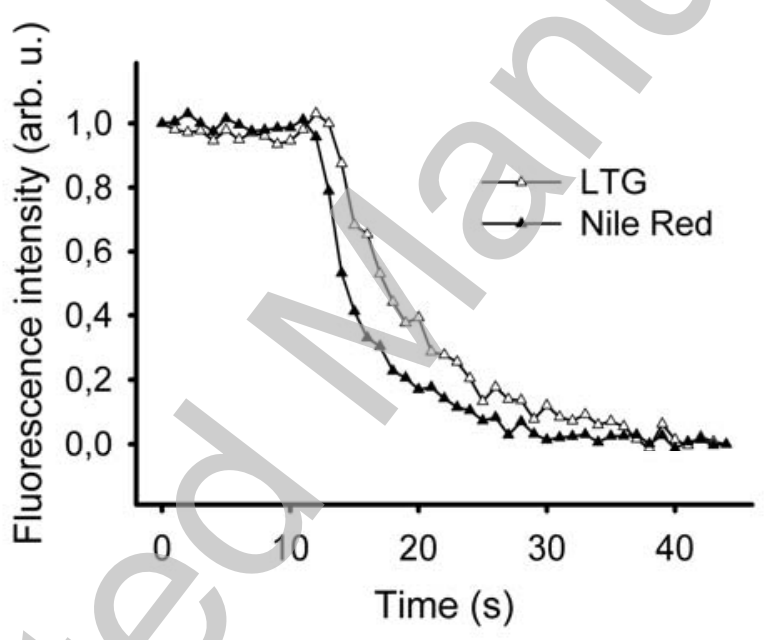

Figure 6: Comparison between content and membrane mixing. A. Co-staining of a single ATII cell with LTG (green) and nile red (red). Acidic vesicles without lipid contents appear green, acidic yesicles with lipid contents (LBs before fusion) appear orange, lipid contents (surfactant) alone (fused LBs) appear red. Scale bar $=10 \mu \mathrm{m}$. B. Time courses of LTG and nile red fluorescence intensities during fusion of a single LB. The average delay between the onsets of fluorescence changes was 1.36 seconds $\pm 0.8(\mathrm{n}=7)$. 

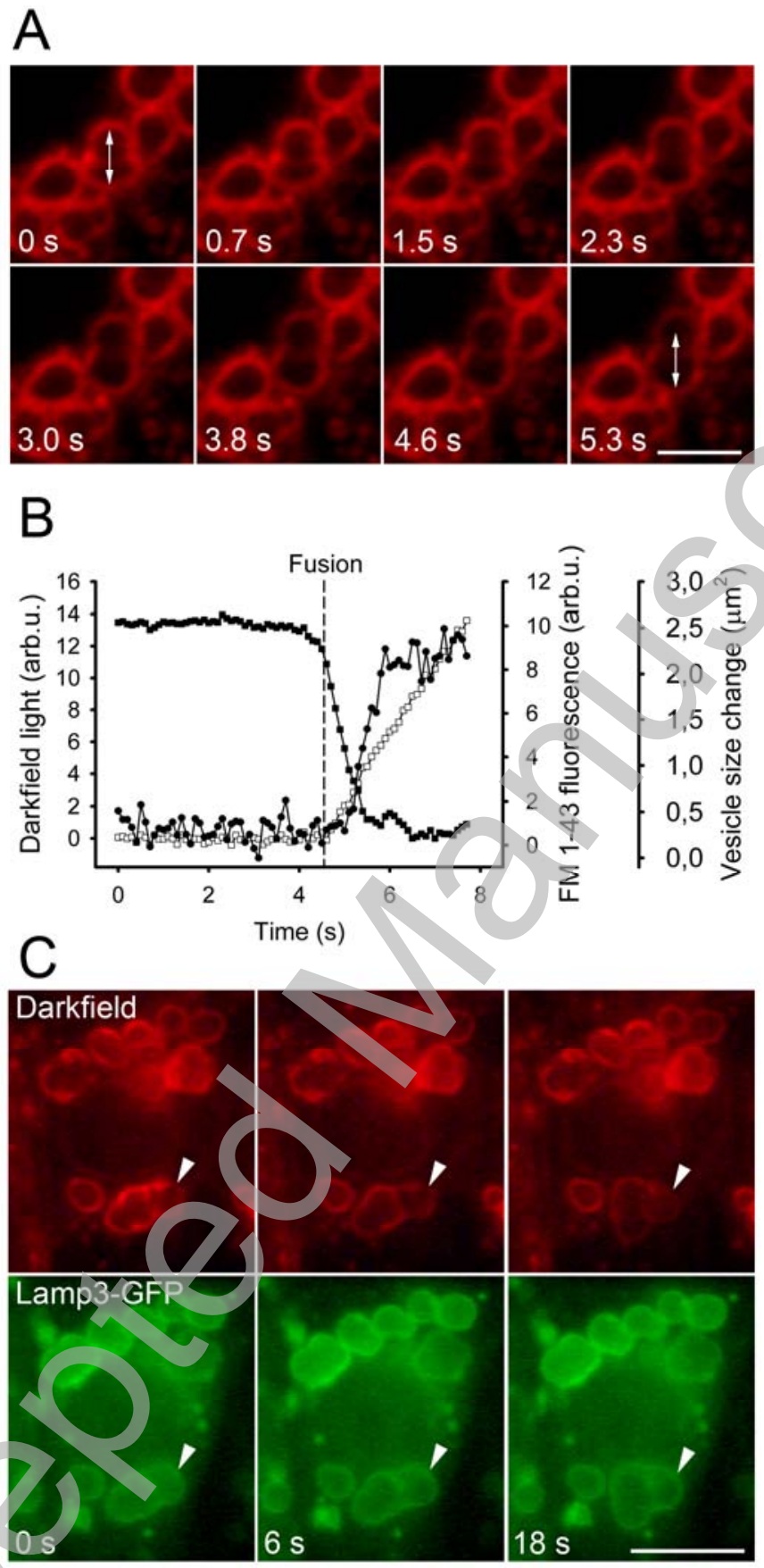

Figure 7: Fusion pore formation was followed by LB swelling. A: Darkfield image series of several LBs, one fusing with the plasma membrane (arrowhead). Time $=0 \mathrm{~s}$ indicates last image before SLID. Double arrow marks initial LB size. Scale bar $=2 \mu \mathrm{m}$. B: Time courses of darkfield light intensity (filled squares), $\mathrm{F}_{\mathrm{FM1}-43}$ (open squares) and vesicle size (filled circles). Image acquisition rate was 10/s. C: LB swelling in ATII cell transfected with the lysosomal vesicle membrane marker Lamp3-GFP. The swelling of the vesicle, observed under darkfield illumination (arrowheads; upper row), was independently 
visible also in Lamp3-GFP fluorescence (arrowheads; bottom row). For additional material see supplementary data Figure 2. Scale bar $=8 \mu \mathrm{m}$. 